\title{
Managing Indigenous Knowledge for Corrective and Preventive Cares: The Case of Horro Guduru Wollega Zone, Oromia, Ethiopia
}

\author{
Hambisa Mitiku1, Worku Jimma ${ }^{2,3, *}$, Chala Diriba ${ }^{3}$ \\ ${ }^{1}$ Department of Information Science, Faculty of Engineering and Technology, Assosa University, Ethiopia \\ ${ }^{2}$ Allied Medical Sciences School, Tehran University of Medical Sciences, Iran \\ ${ }^{3}$ Department of Information Science, College of Natural Sciences, Jimma University, Ethiopia
}

Copyright $\bigcirc 2016$ by authors, all rights reserved. Authors agree that this article remains permanently open access under the terms of the Creative Commons Attribution License 4.0 international License.

\begin{abstract}
This study aimed to investigate the extent to which knowledge management approaches can be used to manage indigenous knowledge (IK) of corrective and preventive cares in local communities of Horro Guduru Wollega zone. This study used descriptive research design through qualitative research method to collect reliable data about perception of disease and its main cause, the types of IK of traditional healthcare practice, indigenous self-medication and required from traditional healthcare practitioners. The respondents were chosen from local communities (traditional healthcare practitioners and community members) and stakeholders (Health Bureau, Agricultural Bureau and NGO). Accordingly, 84 key respondents were chosen through purposive sampling. The qualitative data was analyzed using thematic content analysis. The analysis results have shown that, people in the local community conceptualize disease as the disequilibrium between body and spirit; whereas God, ancestral spirit and witches and sorcerers are the main cause of disease. The above cause's related diseases can be treated by using traditional healthcare IK such as by traditional medicine, physiotherapy treatment and spiritually treatment by self-medication and traditional healthcare practitioners. Therefore, there is a need to capture and manage IK of traditional healthcare practices to save it from loss by local community, community leader and elders, young generations, governments, higher education institutions and researchers.
\end{abstract}

Keywords Indigenous Knowledge, Traditional Healthcare Practices, Self-medication, Corrective Care, Preventive Care

\section{Introduction}

Indigenous Knowledge (IK) is mainly practiced by rural communities in developing countries as a basis for local level decision-making in day-to-day activities of society like in agriculture, health care, education, natural-resource management [1]. Due to the absence of health facilities, people living in rural areas are using indigenous plants as medicines from long ago because this knowledge reaches them through experiences of parental generations [2]. According to WHO [2], proficient medicine by Yoruba tribe in Nigeria consists of knowing the nature of the disease as well as the things that will cause it to go away, thus healing the sick. Iroegbu [3] reported that $80 \%$ of the Nigerian population still relies on indigenous medicine to meet their health-care needs.

According to Cavender [4], IK of traditional healthcare practices is widely accepted as traditional medicine. This method of health care developed out of people's perception of health, response to health care needs and access to health care facilities. IK of traditional healthcare refers to medical knowledge developed by indigenous cultures that incorporates plant, animal and mineral-based medicines, spiritual therapies and manual techniques designed to treat illness or maintain wellbeing [5]. According to Endashaw [6], the use of medicinal plants is as old as human civilization; in that, Ethiopia has glorious tradition of health care system based on plants which dates back to several millennia.

To enhance the benefits that IK can provide in health care, there is a need to explicitly manage IK [7]. To manage IK more efficiently, some Indigenous knowledge System (IKS) have emphasized the development of a holistic approach namely knowledge management (KM) approach, with its theories, principles and practices [8].

Many countries adopted unorthodox measures such as employment of traditional healthcare practices by using their own IK to fulfill their health care needs due to lack of adequate resources [9]. Despite little evidence about the effectiveness of traditional healthcare practices, many 
peoples in the world like in Africa, Asia and Latin America explored possibilities for developing their well-known and tested traditional healthcare practices for use in health care from locally produced materials to meet their health care needs. By this, most of the people are knowledgeable about the administration of a variety of traditional healthcare practices for corrective and preventive purposes. The safety and effectiveness of the traditional healthcare practices is guaranteed by people's knowledge of the main health problems treated and the success in their treatment [6].

Despite the IK potential in traditional healthcare practices, mostly IK is not available in document form because the transfer of IK is through oral traditions and demonstrations among generations, the wide spread of new religion and unevenly sharing of IK in the communities due to issues related to power (considered knowledge as a public good) and cultural differences and this confront IK with irreversible loss [10]. Lack of a cohesive approach for managing IK in developing countries affects the ability of individuals to take advantage of generally available indigenous skills to improve their traditional healthcare practices [11]. Thus, it is important to determine an approach/strategy for managing IK of traditional healthcare practices before much of it is completely lost as KM approach plays a great role in managing IK; in that, it supports people from taking advantage of their skills and innovations in improving IK of traditional healthcare practices in the rural communities of Horro Guduru Wollega zone, Oromia, Ethiopia.

\subsection{Objectives of the Study}

The objectives of this study were:

1. To explore and describe perceptions on disease and its main cause.

2. To identify the types of IK of corrective and preventive cares in the local communities and

3. To describe indigenous corrective and preventive care by self-medication and provided by traditional healthcare practitioners amongst people in the study communities.

\section{Review of Related Literature}

Indigenous knowledge is a know-how, skills and practices, beliefs, representing generations' of experiences, careful observations, trial and error experiments and the product of native people's direct experience with nature and its symbiotic relationship with the social world and is crucial for community survival [12]. The observations made by Dixon [13] in Ethiopia, Kajembe and Kessy [14] in Tanzania and Mudege [15] in Zimbabwe on IK represented as IK is socially and culturally constructed in the local communities. According to Correa [16], IK is the base for local-level decision-making in agriculture, healthcare, food preparation, education, natural resource management and a host of other activities, especially in rural communities. For example, the world market for herbal medicines has reached US $\$ 43$ billion, with annual growth rate of between 5 and $15 \%$, which highlights the importance of IK for socio-economic development.

IK of healthcare is widely accepted as traditional medicine or traditional healthcare practices that are developed out of people's perception of health, response to health care needs and access to health care facilities [17]. Traditional healthcare practice refers to the performance of functions, activities, processes or services based on a traditional philosophy that includes the utilization of traditional practice [18]. Traditional medicine is defined in the traditional health practitioners Act, Act 35 of 2007, as "an object or substance used in traditional health practice for the diagnosis, treatment or prevention of any healing purpose including the maintenance or restoration of physical or mental health or well-being in human beings" whereas traditional healthcare therapies include medication therapies that involve the use of herbal medicines, animal parts and/or minerals and non-medication therapies if they are carried out primarily without the use of medication like manual therapies and spiritual therapies [19].

Regarding the use of traditional healthcare therapies, people identify the disease and its main cause to provide a holistic treatment from traditional healthcare therapies. According to Sargent and Johnson [20], disease is common to all societies, but differs in a way people conceptualize and treat them. By this, disease is a perspective of sickness that refers to some bio-physiological abnormality. In most African countries like Congo, Nigeria, Rwanda and South Africa, diseases are attributed to natural and supernatural diseases (culture syndrome disease) in which natural disease causes includes, adverse climatic conditions, contaminated food and water and the process of ageing and supernatural causes includes, Supreme Being, witchcraft and sorcery and ancestral dissatisfaction [21]. Natural diseases happen spontaneously and are not sent or made to happen. Therefore, it is believed that they do not last very long nor do they return very often and treated by using self-medication [22].

Self-medication is defined by the World Self-Medication Industry (WSMI) as the treatment of common health problems with medicines especially designed and labeled for use without medical supervision and approved as safe and effective for such use. It is common for individuals to feel unwell at one time or the other and the innate survival instinct in humans' produces a tendency to treat them-selves by using home-made materials. Traditional healers with their herbal remedies divination treat both natural and supernatural diseases [23].

It is an established fact that those in traditional African communities, medicinal preparations developed from indigenous medicinal plant species have found application in the management of a wide range of degenerative medical conditions and diseases with culture that is informed by a specific set of cultural beliefs and practices [24]. However, 
due to the prevailing socio-economic and political dynamics worldwide, Western-trained scientists and medical practitioners on the African continent have yet to take up the challenge to conduct large-scale validation of these medicinal entities through internationally recognized scientific methodologies and protocols. In this period, most of the Western-trained African academics and medical experts in South Africa lack an understanding of the economics and the utilization of scientifically approved medication derived from traditional medicine and how it stands to benefit rural economic development; and so far, none of the South African institutions of higher learning offer curricula on IKS [24].

\section{Research Methodology}

For this study, descriptive research design was used through qualitative research method.

\subsection{Methods of Data Collection}

The study was conducted in two districts of rural communities of Horro Guduru Wollega zone namely Jimma Rare and Jimma Horro districts which are selected based on much coverage of the forest used for traditional healthcare (including protected areas, reserves and separated fragments), though not necessarily to sample in every spatial part of the forest. From Jimma Rare district; Dile Kolba and Beda Worke kebele, i.e.,the lowest administrative level in Ethiopia and from Jimma Horro district; Gembo and Mekanesa kebeles were purposely selected based on their farness from health center, lack of health professionals and adoption of traditional healthcare practices.

The study populations are traditional healthcare practitioners, the community members and other stakeholders such as Non-Governmental Organization (NGO), Health bureau and Agricultural bureau. Since IK is unevenly distributed in the local communities [25]; purposive sampling technique was used to select a total of eighty four (84) respondents from the local communities. Focus group discussion, semi-structured interviews and observations were data collections techniques to collect information about IK corrective and preventive cares in the study area. In order to select the respondents, the researchers got a letters from Jimma University and then communicated with the local authorities of the study area. The research objectives were explained to local authorities and permission to conduct research was granted. Moreover, the researchers presented documentation about themselves and the objective of the research before data collection in the field. During the research process, the researchers ensured that, all respondents were properly briefed about the aim of the study, their rights and roles in the study. The respondents were made aware that participation in the study was voluntary and that they were free to withdraw from the discussion at any time.

Accordingly, the researchers have selected 20 traditional healthcare practitioners (five from each kebele), 40 community members (10 from each kebele) and 24 stake holders being with the local authorities. After that, the researchers tried to make some strategies such as planning the work to do, paying money for them as a cash and paying for others as well as inviting them coffee, tea or soft drinks to make the discussion attractive. Accordingly, in each kebele, one focus group discussion was held with traditional healthcare practitioners in whom there are five in number for about two hour, semi-structured interviews with 40 community members and 24 stakeholders for about one hour per each group. Moreover, the key respondents were observed during ritual healing sessions.

\subsection{Data Analysis Procedures}

Qualitative data was analyzed by using thematic content analysis. Analysis were done by producing themes such as indigenous description of diseases, main causes of diseases, indigenous healthcare practices, indigenous mechanisms used for preventive and protective care, corrective care by self-medication and sought from traditional health practitioners

\section{Result and Discussion}

\subsection{Perception and Description of Disease}

All respondents, 84 (100\%) understood human existential conditions as humans are made up of three attributes; namely: physical body (qaama dhaabbataa), spirit (afuura) and personality (namummaa). The physical body decomposes at death after spirit has dead. The spirit was described as a total life. Personality was described as an individuals' celebrity. One's personality can be strong or light depending up on his/her physical body makeup. If one's personality is light, he/she is vulnerable to any disease and bad luck. Good health was understood as a balance between the body and spirit. Most of the respondents, 52 $(62 \%)$ think, disease was considered as a state of disequilibrium between the body and spirit. The understanding of the human conditions is the basis of the type of holistic treatment that involves prevention and corrective care they offer to patients. However, 32 (38\%) of the respondents describe disease as physiological abnormality (dis-functionality of any body part such as liver, eyes, nose, lunges, heart or kidney may result in malfunctioning of the body and body systems such as urinary infections, heart disease and physical disability). Certain body symptoms such as feeling painful, cough, stomach pain and loss of appetite, difficulty in breathing, mental illness and depression, weakened spirit and ritual defilement are indications of particular diseases.

A similar observation was made by Pender and Nola [26], 
understanding of the human existential conditions and perceptions of disease and health enable people to prescribe therapy suitable for the disease. Respondents understanding of phenomena and conditions that are responsible for disease, enable them to seek the correct therapy for the diseases and symptoms they experience and observe. Such perceptions fall within the framework of medical anthropology [27].

\subsection{Main Causes of Diseases}

Sixty one percent $(61 \%)$ of the respondents said the causes of diseases include "God sends a disease as a plague", "ancestral spirits causes disease" and witches and sorcerers brings illness to an individual's. Moreover, 33 (39\%) of the respondents mentioned ecological factors such as climatic conditions and biological and psychological abnormalities such as heredity, accidents and stress as causes of diseases.

\subsection{Corrective Care by Self-medication}

As the data collected from the respondents indicated, $84(100 \%)$ of them define self-medication as giving home-made treatment by using simple household products to cure naturally occurring diseases or symptoms of disease perceived and observed by the patient or by family members. In addition they added that diseases are classified as natural such as cough, flu, blotting, diarrhea, snakebites, fever, hemorrhoids and faintness are always treated by self-medication by applying simple household products such as lemon, orange, salt, cooking oil, ginger, garlic and some herbs.

\subsection{Preventive Care by Self-medication}

All of the respondents, $84(100 \%)$ believed that, promotion of health is achieved through cultural belief systems and practices such as cosmological factors, disease etiologies and isolation and cultural values such as culture, taboos and moral behaviors. In the indigenous cosmology of the study, good health is a balance between the natural environment and human society. Sixty eight (68\%) of the respondents said that understanding elements including change in weather conditions that predisposes peoples to disease and having knowledge about seasonal changes in the weather conditions and about most common diseases that attack people during certain seasons is a helpful mechanism in minimizing chances to contract disease. Accordingly, the people in the community of the study are well aware that some plant species cause disease like body wound or skin diseases and thus avoid contact with such plants.

Half of the respondents (50\%) believes that, disease etiologies such as ancestral spirits, witches and sorcerers, defilement and natural phenomena provide for a number of elements that are causes for disease. Avoidance of disease is maintained through the offering of sacrifices to ones' ancestral spirits, protection of oneself against witchcraft and sorcery and observance of cultural taboos to avoid defilement. In addition, certain diseases such as common cold, whooping cough, diarrhea and scabies are contagious and therefore, in order to avoid transmitting to others, a person who got a contagious disease should be isolated from the community and limit contact with the rest of the family.

All the respondents, $84(100 \%)$ believed that, indigenous mechanisms of preventive healthcare are learnt during enculturation (the process of educating children about cultural values). Indigenous mechanisms of preventive healthcare such as behaviours, attitudes, beliefs and cultural values about the correct behavior for preventing ill health is learnt through informal education in the households through observance of a sick member of the family, taking part in the healing rituals and through questions and folklore.

All the respondents, $84(100 \%)$ believed that, particular cultural restrictions in this community include prohibitions on sexual intercourse with a menstruating woman, a woman who has just got miscarriage, widow and avoidance of contact with polluting objects, people or places promote healthy living. Diseases such as persistent stomach pain, headache and swelling of the stomach resulting from failure to observe cultural restrictions are associated with ritual defilement.

All the respondents, $84(100 \%)$ believed that, good behavior ensures harmonious co-existence and better health; but, God or ancestral spirits punish the breaking of behavioral rules and taboos through disease such as misfortune, infertility, mental illness, physical deformity and unemployment and these are attributed to disrespect, disobedient, neglect and bad luck punishment. Conflicts and influence with neighbors and unfamiliar person are often avoided for fear of witchcraft and sorcery which might result in hated and distrust, stressing situations and diseases such as mental illness and depression.

\subsection{Indigenous Corrective Care by Traditional Healthcare Practitioners}

According to $60(71 \%)$ of respondents, traditional healthcare practitioners' corrective care usually starts with diagnosis about the cause of disease to know the medicines that should be administered for treatment. Thus, diagnosis of disease comprises a combination of procedures including prediction, observation, case history and evaluation of body symptoms to know the disease, its main cause and what treatment could be offered. Prediction was the principal method of diagnosis employed for ancestral intervention to guide the healer to provide information about the origin and nature of the disease and treatment thereof. In addition, 60 (71\%) of respondents, traditional healthcare practitioners cure diseases that attack all age groups and genders through medicinal plant parts, ritual and magical healing techniques. Ritual and magical healing is conducted to cleanse and 
strengthen the patients typically by a sacrificial animal i.e. chicken, goat or sheep is killed and particular parts such as hair, bones and skin are magically prepared to provide corrective care to the patient. Blood is used to cleanse ritual defilement and evil spirits. Medicinal plant parts are prepared and administered in different methods according to the type of diseases requiring treatment.

According, to $20(24 \%)$ of the respondents, traditional health care practitioners showed flexibility in their healing practices in that they may refer their patients to any healthcare alternatives if the patients cannot show any improvement after receiving therapy and where the traditional healthcare practitioners are failing with their treatment.

\subsection{Preventive Care Provided by Traditional Healthcare Practitioners}

From the $84(100 \%)$ of the respondents' point of view, protective is used to protect the personality of a person from disease to ensure good health and for protection against witchcraft and sorcery. In addition, according to them, preventive medicine prepared from medicinal plant is usually in the form of powder or a small portion of the root. The powder is mixed with butter or Vaseline which is smeared on all body parts for preventive purpose or mixed with local drink called areke (a strong locally made alcohol drink) and taken orally. The root of medicinal plant is used to protect people from evil spirit and it is carried in the pocket everywhere the person goes. The children are protected from the evil intentions of witches and evil power through magical charms. Several protective medicines known by the healers are prepared and wrapped into a piece of cloth which is sewn and put around the neck of a child or an adult for protection against attack by evil power.

As a means of preventive care, periodically the head of the family organizes the performance of sacrifice usually made when assistance from ancestors is sought for protective purpose. During the ceremony, several magical herbs are mixed together with the blood of a sacrificial animal (usually a goat and sheep). The purpose of the ritual is to strengthen the family members both physically and spiritually so that they are protected from any attack by disease and witchcraft attempts.

All traditional healthcare practitioners, 20 (24\%) revealed that, certain diseases like culture-bound syndromes do not require modern medicine and therefore, corrective care is through administration of traditional healthcare practices. By this, the above corrective and preventive cares, traditional healthcare practitioners treat and defenses all gender and age groups health problems. The treatment process involves either a full ritual with a sacrifice and herbal treatment. Treatment of culture-bound syndromes such as mental illness, curse, witches, and pain in body part, spirit possession, and ritual defilement requires the offering of sacrifices and herbal treatment prescribed by traditional healthcare practitioners.

\section{Conclusions}

This research aimed to find out the extent to which KM approach is used to manage the IK of corrective and preventive cares in Horro Guduru Wollega zone, Oromia, Ethiopia.

It was found that the community in the study area considers disease as the disequilibrium between physical body and spirit; whereas God, ancestral spirit, witches and sorcerers, ecological factor and biological and psychological factors are disease etiologies. To treat diseases' of these etiologies, peoples use three IK of traditional healthcare practices, namely traditional medicine, physiotherapy and spiritually treatment. Peoples applying self-medication by using simple house hold products to treat naturally occurring diseases; but, required traditional healthcare practitioners' if their knowledge of self-medication is failed. It can be concluded that traditional healthcare practices is common in this community and there is a need to capture as well as manage the indigenous knowledge so that it is not lost.

\section{Acknowledgements}

The authors acknowledge Jimma University for the financial support to conduct this research and the Department of Information Science of Jimma University for facilitating the research work by providing us the necessary facilities as well as providing us with support letters to the local authorities of the study area to permit us to carry out the research.

\section{Appendix: A check list used to interview the study participants}

Part I: Describing disease and its main cause

1. How would you describe a disease/illness?

2. What are the main causes of disease in your community?

Part II: Identifying self-medication and corrective care sought from traditional healthcare practitioners.

1. What is meant by self-medication?

2. Which diseases do you cure and prevent by self-medication? How?

3. What are the indigenous mechanisms you would use to prevent being affected by disease/illness?

4. Which indigenous corrective and defensive care is required from traditional health practitioners?

5. Which diseases are cured by traditional health practitioners?

6. What health care facilities are available in your community?

7. Which one do you prefer? Why?

8. What is the traditional health practitioner's consultation fee? 
Is the fee is higher than what you could pay in clinics and hospitals.

\section{REFERENCES}

[1] Warren DM, Slikkerveer LJ, Brokensha D. The cultural dimension of development: indigenous knowledge systems. Intermediate Technology Publications Ltd (ITP); 1995.

[2] WHO (2000), Health Systems: Improving performance, Geneva; Available at http://www.who.int/ (accessed on 4 May 2015).

[3] Iroegbu, E., (2006), Knowledge of Herbal Resources and Development of Practitioners in Nigerian Society, Indilinga: African Journal of Indigenous Knowledge Systems. Vol., 5, No.1; pp. 32-49.

[4] Cavender, A., (1991), Traditional medicine and an inclusive model of health seeking behavior in Zimbabwe: Central African Journal of Medicine, Vol. 37, pp. 362 - 369.

[5] Courtright, P., (2000), Collaboration with traditional health practitioners for the prevention of blindness, London: World Scientific. Available on the web: www.who.int/ncd/division 2020 action plan/documents/cath.pdf (accessed on 13 April 2015).

[6] Endashaw, B., (2007), Study on Actual Situation on Medicinal Plants in Ethiopia, http://www.endanshaw.com: (accessed 10 Sep 2015).

[7] Mostert, J., and Snyman, M., (2007), Knowledge Management Strategy for the Development of an Effective Knowledge Management Strategy: South African Journalof Information Management, Vol. 9, No.2, pp. 34-36.

[8] Kaniki, A. and Mphahlele, M., (2002), Indigenous knowledge for the benefit of all: can knowledge management principles be used effectively? South African Journal of Library and Information Science, Vol 68, No.1; pp. 67-73.

[9] Dennil, K., (1999) Aspects of primary health care: Community health care in South Africa. Cape Town: Oxford University Press.

[10] Hamilton, A., (2003), Medicinal Plants and Conservation: Issues and Approaches; International Plants Conservation Unit, WWF- UK.

[11] Akiiki, E., (2006), Linking healthcare innovations to knowledge sharing in Africa: IK Notes, vol. 88, pp. 1-3.

[12] Nakashima, Douglas, Galloway McLean, Kirsty, Thulstrup, Hans, Ramos-Castillo, Ameyali and Rubis, Jennifer, Weathering uncertainty: traditional knowledge for climate change assessment and adaptation, (Paris and Darwin, Australia: UNESCO and United Nations University Traditional Knowledge Initiative, 2012).

[13] Dixon, A., (2002) the role of indigenous knowledge in wetland management: mechanisms of knowledge acquisition and development basis for sustainable use; WeNReG Working paper 4 .

[14] Kajembe, G., and Kessy, J., (1999), Evaluation of forestry extension services of herbal medicine in Mwanza and Tabora regions, Tanzania. A consultancy report submitted to the Ministry of Natural Resources and Tourism, Tanzania.

[15] Mudege, N., (2005), Knowledge production and dissemination in land resettlement areas in Zimbabwe: the case of Mupfurudzi. Ph.D. thesis; Wageningen: Wageningen University.

[16] 16. Correa, C., (2001), Traditional knowledge and intellectual property: issues and options surrounding the protection of traditional knowledge, 12 October 2001.

[17] Hjelm, (1999), Beliefs about health and illness essential for self-care practice: a comparison of migrant Yugoslavian and Swedish diabetic females; Journal of Advanced Nursing, Vol. 30, No.5, pp.1147-59.

[18] Truter, I. (2007), African Traditional Healers: Cultural and Religious Belief intertwined in a historic way; South African Pharmaceutical Journal, Vol.7, pp. 83-89.

[19] WHO (2002), Human health and dams: Report submitted to the World Commission on Dams; WHO/SDE/WSH/00.01g

[20] Sargent, C. and Johnson, T (1996), Medical anthropology: Contemporary theory and method. London: Praeger.

[21] Peek, K., (1991), African divination systems, Bloomington and Indianapolis: Indiana university press.

[22] Kriel, D., (1992), Die Siektebegrip van die Noord-sotho: unpublished D.Phil thesis Pretoria, university of Pretoria.

[23] Pelzer, K., (1998), A community survey traditional healers in south Africa (Northern province), South African journal of ethnology, Vol.21, No. 4; pp. 191-97.

[24] Posey, A., (1999), Cultural and Spiritual Value of Biodiversity: A Complementary Contribution to the Global Biodiversity Assessment, London: Intermediate Technology Publications.

[25] IIRR (1996), Recording and Using Indigenous Knowledge: A manual, International Institute for Rural Reconstruction (IIRR), Silang, and Cavite, Philippines.

[26] Pender, L. and Nola, S., (2005), The health promotion model: St. Louis: Mosby.

[27] Sargent, C. and Johnson, T (1996), Medical anthropology: Contemporary theory and method. London: Praeger. 